ARTICLE

\title{
Superconductivity emerging from a suppressed large magnetoresistant state in tungsten ditelluride
}

\author{
Defen Kang ${ }^{1, \star}$, Yazhou Zhou ${ }^{1, \star}$, Wei $\mathrm{Yi}^{1}$, Chongli Yang ${ }^{1}$, Jing Guo ${ }^{1}$, Youguo Shi ${ }^{1}$, Shan Zhang ${ }^{1}$, \\ Zhe Wang ${ }^{1}$, Chao Zhang' ${ }^{1}$ Sheng Jiang ${ }^{2}$, Aiguo Li $^{2}$, Ke Yang ${ }^{2}$, Qi Wu', Guangming Zhang ${ }^{3,4}$, \\ Liling Sun ${ }^{1,4} \&$ Zhongxian Zhao ${ }^{1,4}$
}

The recent discovery of large magnetoresistance in tungsten ditelluride provides a unique playground to find new phenomena and significant perspective for potential applications. The large magnetoresistance effect originates from a perfect balance of hole and electron carriers, which is sensitive to external pressure. Here we report the suppression of the large magnetoresistance and emergence of superconductivity in pressurized tungsten ditelluride via high-pressure synchrotron X-ray diffraction, electrical resistance, magnetoresistance and alternating current magnetic susceptibility measurements. Upon increasing pressure, the positive large magnetoresistance effect is gradually suppressed and turned off at a critical pressure of $10.5 \mathrm{GPa}$, where superconductivity accordingly emerges. No structural phase transition is observed under the pressure investigated. In situ high-pressure Hall coefficient measurements at low temperatures demonstrate that elevating pressure decreases the population of hole carriers but increases that of the electron ones. Significantly, at the critical pressure, a sign change of the Hall coefficient is observed.

\footnotetext{
${ }^{1}$ Institute of Physics and Beijing National Laboratory for Condensed Matter Physics, Chinese Academy of Sciences, Beijing 100190, China. ${ }^{2}$ Shanghai Synchrotron Radiation Facilities, Shanghai Institute of Applied Physics, Chinese Academy of Sciences, Shanghai 201204, China. ${ }^{3}$ State Key Laboratory for Low dimensional Quantum Physics, Department of Physics, Tsinghua University, Beijing 100084, China. ${ }^{4}$ Collaborative Innovation Center of Quantum Matter, Beijing 100190, China. ${ }^{\star}$ These authors contributed equally to this work. Correspondence and requests for materials should be addressed to L.S. (email: Ilsun@iphy.ac.cn) or to Z.Z. (email: zhxzhao@iphy.ac.cn).
} 
$\mathrm{T}$ ungsten ditelluride $\left(\mathrm{WTe}_{2}\right)$ is a well-known non-magnetically thermoelectric semimetal ${ }^{1-3}$. Recently, its unexpected property of large magnetoresistance (LMR) has been discovered ${ }^{4}$, yielding a research hotspot in condensed matter physics and material science. The LMR effect found in $\mathrm{WTe}_{2}$ and some other nonmagnetic compounds ${ }^{4-10}$ is a peculiar transport property. At ambient pressure, $\mathrm{WTe}_{2}$ crystallizes in a crystal structure of distorted $\mathrm{MoS}_{2}$ (ref. 11), and the distortion is induced by the tungsten chains that are arranged along the $a$ axis of the orthorhombic unit cell. The electrical resistance along the $a$ axis increases markedly when magnetic field is applied perpendicularly to the dichalcogenide layers (along the $c$ axis), resulting in the LMR effect. Theoretical and experimental investigations indicate that the LMR effect is resulted from the perfect balance between electron and hole Fermi pockets along the $\Gamma-\mathrm{X}$ direction in the Brillouin zone $e^{4,12,13}$, different from that of giant and colossal magnetoresistance previously found in magnetic materials ${ }^{14,15}$. Due to the complicated band structure with multiple Fermi pockets in $\mathrm{WTe}_{2}$, the fine details of the electronic structure play a significant role in the LMR effect. It is well known that pressure as an important control parameter can effectively tune lattice structures and the corresponding electronic states in a more systematic fashion, avoiding the complexity brought by chemical doping ${ }^{13,16-20}$. In particular, the Fermi surface changes had been observed in pressurized $\mathrm{NbSe}_{2}$ and $\mathrm{NbS}_{2}$ (ref. 21 and ref. 22, respectively). Therefore, the highpressure studies on $\mathrm{WTe}_{2}$ are important to explore novel phenomena and understand the physics behind.

We demonstrate here that how external pressure suppresses the LMR state and induces the emergence of superconductivity in $\mathrm{WTe}_{2}$ with the experimental results from multiple in situ highpressure measurements. We find that, upon increasing pressure, the inter-plane lattice constant $c$ is substantially reduced without structural phase transition, while the LMR effect is gradually suppressed and turned off at a critical pressure of $10.5 \mathrm{GPa}$ where superconductivity accordingly emerges. The superconducting transition temperature $\left(T_{\mathrm{c}}\right)$ reaches $6.5 \mathrm{~K}$ at $\sim 13.0 \mathrm{GPa}$ and then decreases monotonically with increasing pressure down to $2.6 \mathrm{~K}$ at $\sim 24.0 \mathrm{GPa}$. Significantly, our Hall measurements reveal a sign change in the Hall coefficient at the critical pressure, indicating a quantum phase transition of the Fermi surface reconstruction.

During the preparation of this manuscript, we became aware of an arXiv paper that reports the evidence of pressure-induced superconductivity in $\mathrm{WTe}_{2}$ from electrical resistance measurements without a pressure-transmitting medium ${ }^{23}$.

\section{Results}

Structure under pressure. We first characterize the structure of the $\mathrm{WTe}_{2}$ sample at ambient pressure. Figure 1a shows the X-ray diffraction (XRD) pattern of a powdered sample that is ground from a few pieces of single crystals. As it can be seen, the Bragg peaks in the pattern can be well indexed by orthorhombic structure. To clarify whether there is a structure change in pressurized $\mathrm{WTe}_{2}$, we perform in situ high-pressure synchrotron XRD measurements. The results shown in Fig. $2 \mathrm{a}$ indicate no first-order structure phase transition under pressure up to $20.1 \mathrm{GPa}$. Then we extract the lattice parameters as a function of pressure, shown in Fig. 2b,c. The pressure dependence of volume also displays in Fig. 2 d. It is found that the lattice constants $(a, b$ and c) as well as the volume decrease continuously upon increasing pressure. However, the reduction of $c$ is substantial, compared with those of the in-plane parameters.

Pressure-induced superconductivity. The electrical resistance measurement for the single crystal is performed under quasi-

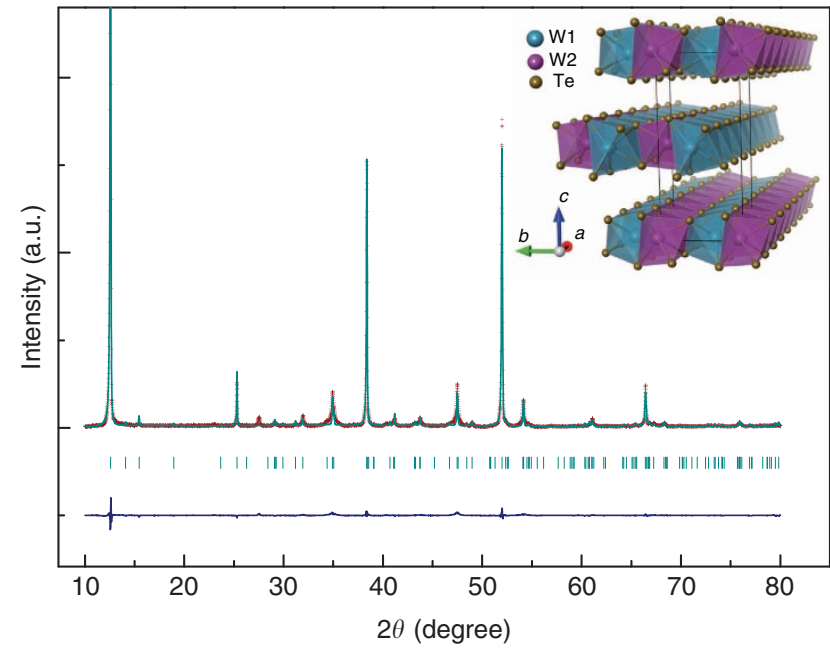

Figure 1 | Experimental and indexed X-ray diffraction patterns of $\mathrm{WTe}_{\mathbf{2}}$ at ambient pressure. The purple crosses represent experimental data, and the cyan line and bars represent calculated Bragg reflection pattern and positions. The inset shows the three-dimensional layer structure constructed by an edge-shared $\mathrm{WTe}_{6}$ octahedron.

hydrostatic pressure. Since $\mathrm{WTe}_{2}$ has an anisotropic electron structure, the LMR effect was discovered along the tungsten chain direction $(a \text { axis })^{4}$. To reveal the pressure effect on the LMR state, we apply the magnetic field and current in the same manner as ambient pressure. Figure 3 shows the typical temperature dependence of electrical resistance measured at zero magnetic field for pressures ranging from 0.3 to $24.0 \mathrm{GPa}$. In Fig. 3a, the electrical resistance curve at $0.3 \mathrm{GPa}$ shares the similar behaviour to that measured at ambient pressure ${ }^{4,13}$. Upon increasing pressure below $13.0 \mathrm{GPa}$, the electrical resistance in the whole temperature range is suppressed, while it is enhanced above $13.0 \mathrm{GPa}$. Intriguingly, at the pressure of $10.5 \mathrm{GPa}$, the resistance drops abruptly at $2.8 \mathrm{~K}$. Such a drop becomes more pronounced at higher pressures, and the zero-electrical resistance is achieved between 11.0 and $24.0 \mathrm{GPa}$ (Fig. 3b).

To confirm whether the zero-electrical resistance state is superconducting or not, we carry out in situ high-pressure alternating-current (a.c.) susceptibility measurements. The Meissner effect is observed at the selected pressures of 15.0 and $18.3 \mathrm{GPa}$, respectively (Fig. 3c). The onset temperatures of the diamagnetism are consistent with that of the electrical resistance drop (Fig. 3b). Both electrical resistance and magnetic measurements coordinately confirm that pressure induces a superconducting transition in $\mathrm{WTe}_{2}$. The $T_{\mathrm{c}}$ can reach to $6.5 \mathrm{~K}$ at $\sim 13.0 \mathrm{GPa}$ and monotonically decreases down to $2.6 \mathrm{~K}$ at $\sim 24.0 \mathrm{GPa}$.

Suppression of the LMR state. To reveal how the LMR state evolves into the superconducting state, we systematically investigate the temperature dependence of electrical resistance at fixed pressures under different magnetic fields. We find that the positive LMR effect of $\mathrm{WTe}_{2}$ is suppressed by applying pressure (Fig. $4 \mathrm{a}, \mathrm{b})$. At the pressure of $11.0 \mathrm{GPa}$ and above, the positive magnetoresistance effect no longer exists (Fig. 4c,d), and the superconductivity appears simultaneously.

Determination of upper critical magnetic field. Notably, the superconductivity of the sample is fully suppressed at $13.0 \mathrm{GPa}$ under 3 and 7 Tesla (Fig. 4d). To determine the value of upper critical magnetic field $\left(H_{\mathrm{c} 2}\right)$ precisely, we perform the temperature 
a

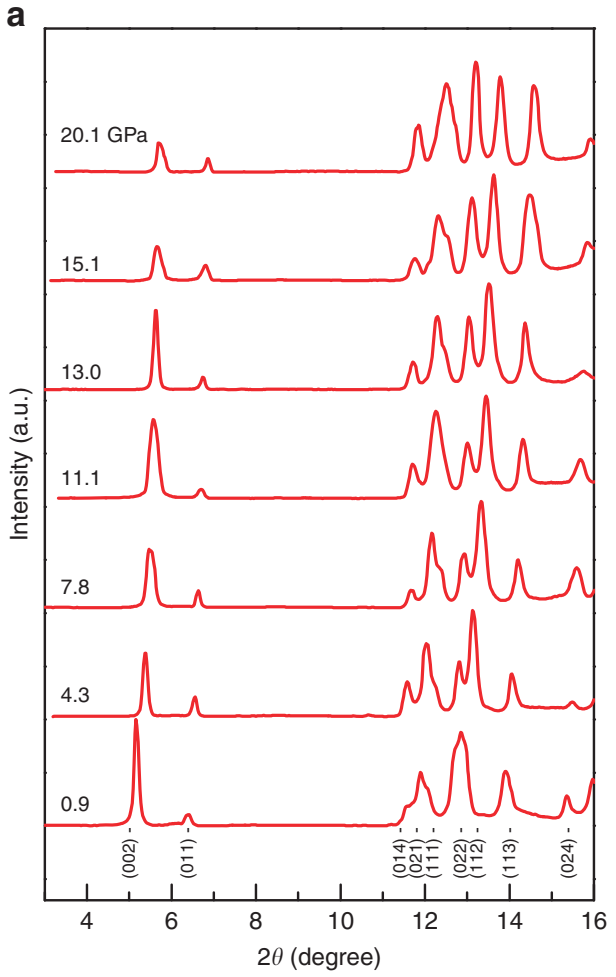

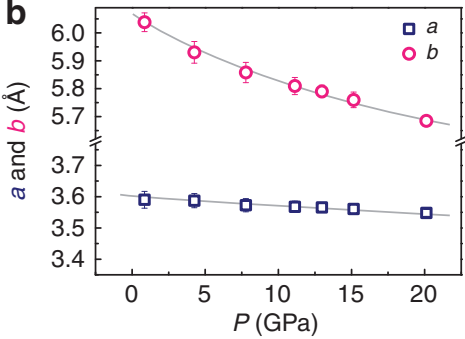
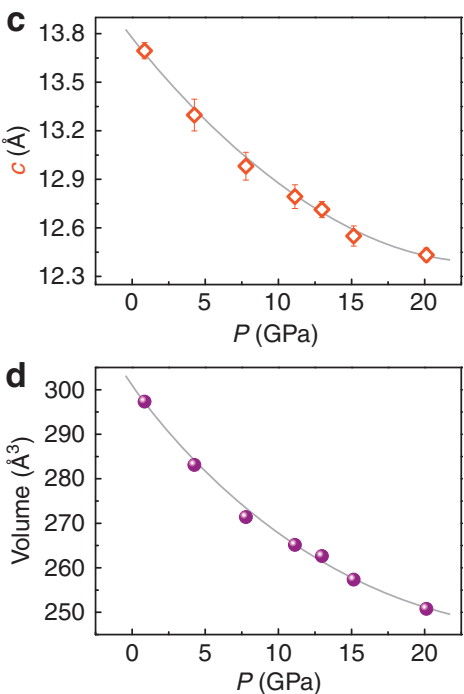

Figure 2 | Structure information of WTe $\mathbf{2}$ sample at high pressure. (a) X-ray diffraction patterns of WTe $\mathrm{W}_{2}$ collected at different pressures, showing no crystal structure transition. (b,c) Pressure dependence of lattice constants $(a, b$ and $c$ ). The error bars represent s.d. (d) Volume change as a function of pressure.
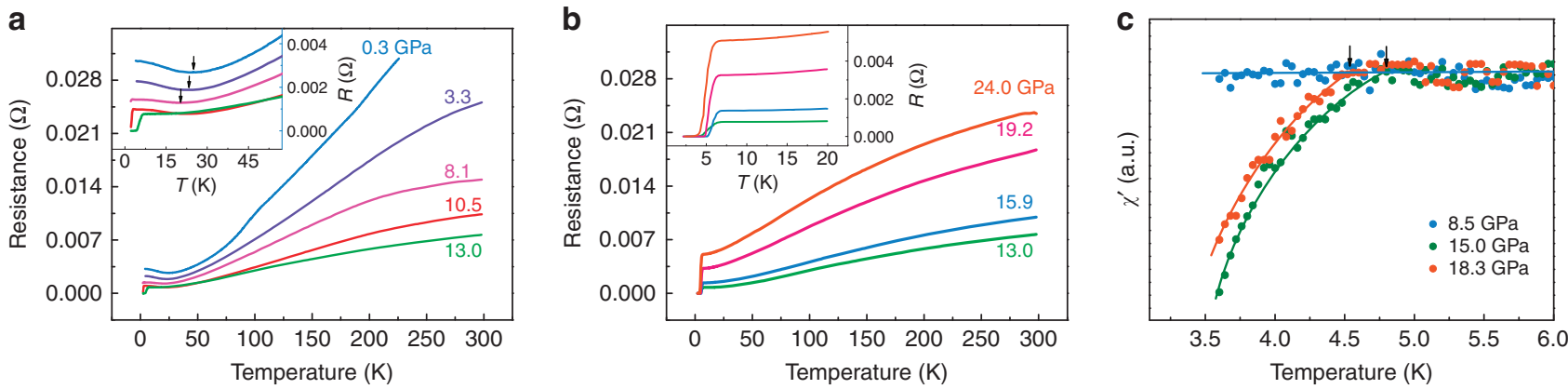

Figure 3 | Electrical resistance and susceptibility features of single-crystal $\mathbf{W} \mathbf{T e}_{\mathbf{2}}$ at different pressures. (a) The plot of electrical resistance as a function of temperature measured without magnetic field for the pressures ranging from 0.3 to $13.0 \mathrm{GPa}$. The inset displays the enlarged view of the lowtemperature electrical resistance, where a characteristic temperature is defined by the minimum resistance and is taken as turn-on temperature ( $T^{\star} \mathrm{ZF}$ ) of the LMR effect, as indicated by arrows. (b) Temperature dependence of electrical resistance measured at different pressures between 13.0 and $24.0 \mathrm{GPa}$. The inset clearly shows electrical resistance drops and zero-resistance behaviour. (c) The real part of the a.c. magnetic susceptibility $\left(\chi^{\prime}\right)$ versus temperature for the single-crystal $\mathrm{WTe}_{2}$ at different pressures, which confirms the diamagnetism at the selected pressures of 15.0 and $18.3 \mathrm{GPa}$, respectively. The arrows indicate the onset temperatures of the superconducting transitions.

dependence of resistance measurements under lower magnetic fields on the pressurized sample (Fig. 5a-c). Using the GinzburgLandau formula to fit the experimental data yields the values of upper critical magnetic field at zero temperature: $1.86 \mathrm{~T}$ at 13.7 $\mathrm{GPa}, 1.44 \mathrm{~T}$ at $16.0 \mathrm{GPa}$ and $0.85 \mathrm{~T}$ at $19.4 \mathrm{GPa}$ (Fig. $5 \mathrm{~d}$ ).

Pressure-temperature phase diagram. A characteristic temperature $\left(T^{*} \mathrm{ZF}\right)$ as the turn-on temperature of the LMR effect at zero field is defined, as indicated by the arrows in the inset of
Fig. 3a. Such a definition of the $T^{*} \mathrm{ZF}$ is coincident with the temperature of the linear extrapolation of turn-on LMR temperatures under different magnetic fields. Then we summarize our experimental results in the pressure-temperature phase diagram (Fig. 6a). There are two distinct regions in the diagram: the LMR state and the superconducting state. It is found that the $T^{\star} \mathrm{ZF}$ of the LMR state decreases with increasing pressure and vanishes at the critical pressure $10.5 \mathrm{GPa}$, where the superconductivity emerges at $2.8 \mathrm{~K}$. The value of $T_{c}$ increases up to a maximum at $13.0 \mathrm{GPa}$ and then declines with further increasing pressure. 

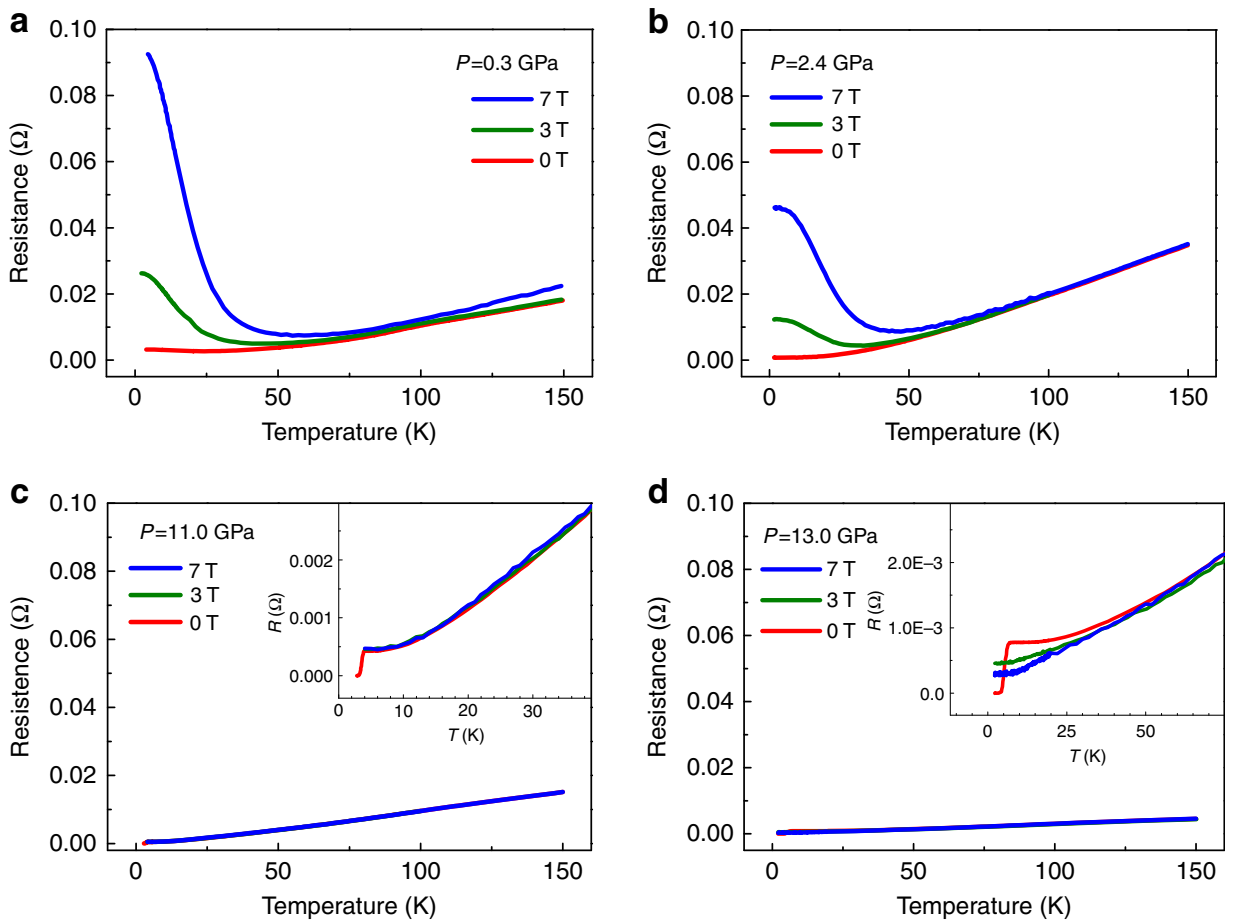

Figure 4 | High-pressure electrical resistance versus temperature under different magnetic fields. (a,b) The electrical resistance as a function of temperature at 0.3 and $2.4 \mathrm{GPa}$, respectively, illustrating the obvious suppression of the LMR effect by increasing pressure. (c) The plot of resistance versus temperature at $11.0 \mathrm{GPa}$. The inset shows an enlarged view of a full suppression of the positive magnetoresistance and a zero resistance at $3.2 \mathrm{~K}$.

(d) The temperature dependence of electrical resistance at $13.0 \mathrm{GPa}$. The inset displays the elimination of superconductivity by magnetic fields.
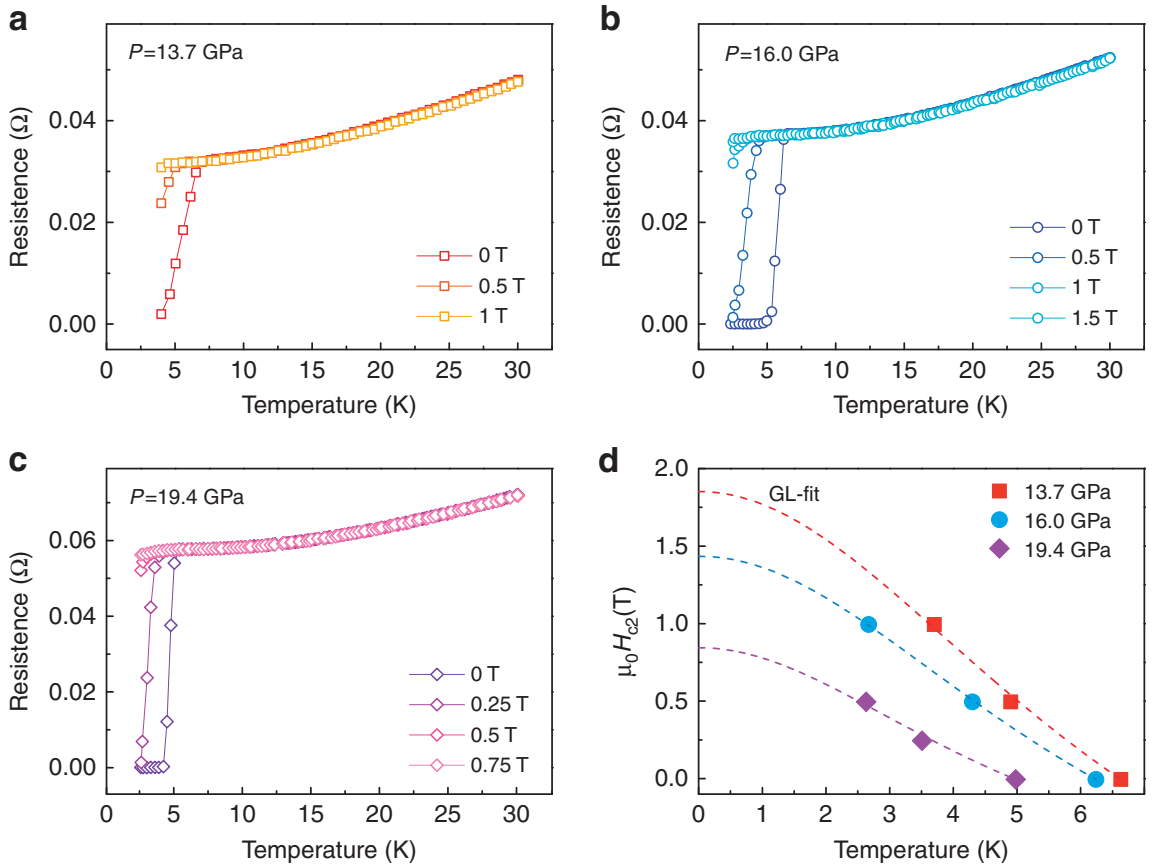

Figure 5 | Determination of the value of upper critical field $\left(\boldsymbol{H}_{\mathbf{c} 2}\right)$ for the superconducting WTe. $\mathbf{~ ( a - c ) ~ T e m p e r a t u r e ~ d e p e n d e n c e ~ o f ~ e l e c t r i c a l ~ r e s i s t a n c e ~}$ at fixed pressures under different magnetic fields. (d) $H_{c 2}$ as a function of temperature. The dashed lines represent the Ginzburg-Landau (GL) fits.

This phase diagram clearly demonstrates how the pressure can effectively suppress the LMR state and induce superconductivity.

Change of Hall coefficient with pressure. It is known that the perfect balance between hole and electron populations accounts for the ambient-pressure LMR effect ${ }^{4,12}$. To understand the suppression of the LMR effect under pressure, we conduct the in situ high-pressure Hall coefficient $\left(R_{\mathrm{H}}\right)$ measurements at low temperature (Fig. 6b). The set-up of our high-pressure Hall measurements leads to the detected $R_{\mathrm{H}}$ combining signals from 


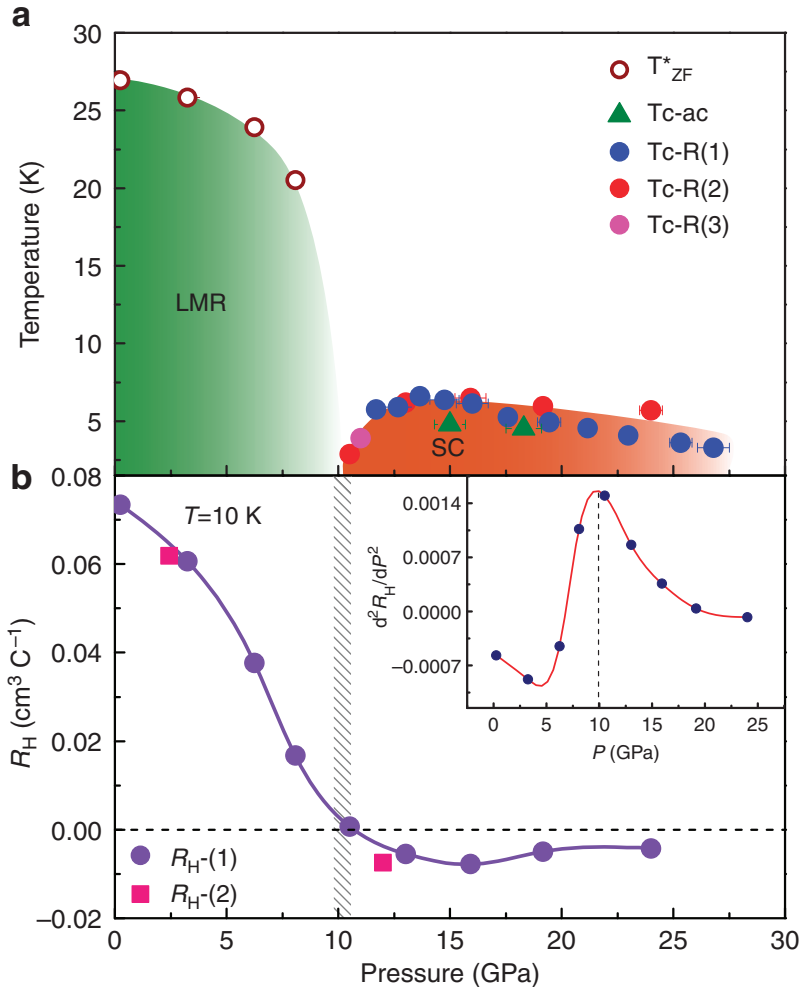

Figure 6 | Pressure-temperature phase diagram of $\mathrm{WTe}_{2}$ and pressuredependent Hall coefficient. (a) The $T^{\star}{ }_{Z F}$ and $T_{c}$ versus pressure. The red, pink and blue solid circles represent $T_{\mathrm{c}}$ extracted from different runs of electrical resistance measurements, and the green triangles represent the $T_{c}$ determined from the a.c. susceptibility measurements. The acronyms LMR and SC stand for the large magnetoresistant state and superconducting state, respectively. The error bars represent the s.d. (b) Hall coefficient $\left(R_{H}\right)$ as a function of pressure measured at $10 \mathrm{~K}$ and 1 Tesla, displaying a sign change from the positive to the negative at the critical pressure of $10.5 \mathrm{GPa}$. Solid purple circles and pink squares represent the $R_{H}$ obtained from different runs. The inset shows the second derivative of the Hall coefficient, the maximum of which corresponds to the sign change of Hall coefficient. The shaded area indicates the pressure range where the superconductivity emerges and the sign of $R_{\mathrm{H}}$ changes.

the two components of parallel and perpendicular to the tungsten chains (see Methods). Thus, the obtained results involve contributions from both hole and electron carries at the Fermi surface in the Brillouin zone. Below $10.5 \mathrm{GPa}$, the $R_{\mathrm{H}}$ is positive and it decreases with elevating pressure. On further increasing pressure, significantly, we find that the $R_{\mathrm{H}}$ suffers from a sign change from the positive to the negative at the critical pressure of 10.5 GPa, where the LMR state vanishes and superconductivity emerges.

\section{Discussion}

The sign change in $R_{\mathrm{H}}$ is an indication of a significant reconstruction of Fermi surface, in good agreement with the reported Shubnikov-de-Hass oscillation measurements ${ }^{13}$. At the critical pressure, the second derivative of the Hall coefficient with respect to pressure shows a maximum, which is expected to become divergent in the zero temperature limit. Actually, our high-pressure XRD measurements indicate that, at the critical pressure, the $c$ axis is compressed by $6.5 \%$, almost 10 -fold of the $a$-axis compressibility $(0.6 \%)$ and twofold of the $b$-axis compressibility (3.3\%). These results suggest that the reconstruction of Fermi surface is associated with anisotropic reductions of the lattice.

We thus propose that a quantum phase transition occurs at the critical pressure, separating the LMR state and superconducting state. Such a kind of quantum phase transition with the changes of the Fermi surface structure can be characterized by the Lifshitz phase transition ${ }^{24}$, so the emergence of superconductivity observed in the pressurized $\mathrm{WTe}_{2}$ may be connected with this transition, reminiscent of what is seen in Fe-based superconductors $^{25}$. The mechanism of superconductivity in $\mathrm{WTe}_{2}$ deserves further investigations from both experimental and theoretical sides.

\section{Methods}

Single-crystal growth. Single crystals of $\mathrm{WTe}_{2}$ were grown by means of a solidstate reaction. Tungsten powder (99.9\%) was mixed with excessive amounts of tellurium (99.999\%) and placed in an alumina ampoule, then sealed in an evacuated quartz tube. The operation above was performed in a glove box filled with high-purity argon gas. The mixture was heated in a furnace to $1,000{ }^{\circ} \mathrm{C}$ with a rate of $100^{\circ} \mathrm{Ch}^{-1}$, followed by keeping the temperature at $1,000^{\circ} \mathrm{C}$ for $5 \mathrm{~h}$. After reaction, the sample was cooled down to $800^{\circ} \mathrm{C}$ at a rate of $1^{\circ} \mathrm{Ch}{ }^{-1}$, then to $700{ }^{\circ} \mathrm{C}$ at a rate of $5^{\circ} \mathrm{C} \mathrm{h}^{-1}$. The quartz tube was then taken out from the furnace, and put in a centrifuge to remove the Te flux.

Experimental details of high-pressure measurements. Pressure was generated by a device, the so-called diamond anvil cell that consists of two opposing anvils sitting on the supporting plates. Diamond anvils of $300-\mu \mathrm{m}$ flats were used for this study. Nonmagnetic rhenium gaskets with a $100-\mu \mathrm{m}$ diameter hole were used for different runs of the high-pressure studies. The four-probe method was applied in the $a b$ plane of the single-crystal $\mathrm{WTe}_{2}$ for all high-pressure transport measurements. For the high-pressure Hall coefficient measurements, the van der Pauw method was applied in this study. A constant current goes through the squared sample diagonally, and the Hall voltage is measured from the other diagonal. To keep the sample in a quasi-hydrostatic pressure environment, $\mathrm{NaCl}$ powder was employed as the pressure medium. The high-pressure alternating-current susceptibilities were detected using a primary/secondary-compensated coil system surrounding the sample ${ }^{26}$. High-pressure XRD experiments were performed at beam line $15 \mathrm{U}$ at the Shanghai Synchrotron Radiation Facility. A monochromatic X-ray beam with a wavelength of $0.6199 \AA$ was chosen for all XRD measurements. Diamonds with low birefringence were selected for the experiments. To maintain the sample in a hydrostatic pressure environment, silicon oil was used as a pressure medium in the high-pressure XRD measurements. Pressure was determined by the ruby fluorescence method $^{27}$.

\section{References}

1. Callanan, J. E., Hope, G. A., Weir, R. D. \& Westrum, E. F. Thermodynamic properties of tungsten ditelluride $\left(\mathrm{WTe}_{2}\right) \mathrm{I}$. The preparation and low temperature heat capacity at temperatures from $6 \mathrm{~K}$ to $326 \mathrm{~K}$. J. Chem. Thermodyn. 24, 627-638 (1992).

2. Kabashim, S. Electrical properties of tungsten-ditelluride WTe 2 . J. Phys. Soc. Jpn 21, 945 (1966).

3. Revolinsky, E. \& Beerntsen, D. Electrical properties of the $\mathrm{MoTe}_{2}-\mathrm{WTe}_{2}$ and $\mathrm{MoSe}_{2}-\mathrm{WSe}_{2}$ systems. J. Appl. Phys. 35, 2086-2089 (1964).

4. Ali, M. N. et al. Large, non-saturating magnetoresistance in $\mathrm{WTe}_{2}$. Nature 514, 205-208 (2014).

5. Liang, T. et al. Ultrahigh mobility and giant magnetoresistance in the Dirac semimetal $\mathrm{Cd}_{3} \mathrm{As}_{2}$. Nat. Mater. 14, 280-284 (2014).

6. Mun, E. et al. Magnetic field effects on transport properties of $\mathrm{PtSn}_{4}$. Phys. Rev. $B$ 85, 035135 (2012).

7. Wang, K., Graf, D., Li, L., Wang, L. \& Petrovic, C. Anisotropic giant magnetoresistance in $\mathrm{NbSb}_{2}$. Sci. Rep. 4, 7328 (2014).

8. Galvis, J. A., Suderow, H., Vieira, S., Bud'ko, S. L. \& Canfield, P. C. Scanning tunneling microscopy in the superconductor $\mathrm{LaSb}_{2}$. Phys. Rev. B 87, 214504 (2013).

9. Young, D. P. et al. High magnetic field sensor using LaSb ${ }_{2}$. Appl. Phys. Lett. 82, 3713-3715 (2003).

10. Bud'ko, S. L., Canfield, P. C., Mielke, C. H. \& Lacerda, A. H. Anisotropic magnetic properties of light rare-earth diantimonides. Phys. Rev. B 57, 13624 (1998).

11. Brown, B. E. The crystal structures of $\mathrm{WTe}_{2}$ and high-temperature $\mathrm{MoTe}_{2}$. Acta Crystallogr. 20, 268-274 (1966).

12. Pletikosic, I., Ali, M. N., Fedorov, A. V., Cava, R. J. \& Valla, T. Electronic structure basis for the extraordinary magnetoresistance in $\mathrm{WTe}_{2}$. Phys. Rev. Lett. 113, 216601 (2014).

13. Cai, P. L. et al.Drastic pressure effect on the extremely large magnetoresistance in $\mathrm{WTe}_{2}$. Preprint at http://arxiv.org/abs/1412.8298 (2014). 
14. Baibich, M. N. et al. Giant magnetoresistance of (001)Fe/(001)Cr magnetic superlattices. Phys. Rev. Lett. 61, 2472-2475 (1988).

15. Salamon, M. B. \& Jaime, M. The physics of manganites: Structure and transport. Rev. Mod. Phys. 73, 583-628 (2001).

16. Chi, Z.-H. et al. Pressure-induced metallization of molybdenum disulfide. Phys. Rev. Lett. 113, 036802 (2014).

17. Drozdov, A. P., Eremets, M. I. \& Troyan, I. A. Conventional superconductivity at $190 \mathrm{~K}$ at high pressures. Preprint at http://arxiv.org/abs/1412.0460 (2014).

18. Mackenzie, A. P. \& Grigera, S. A. A quantum critical route to field-induced superconductivity. Science 309, 1330-1331 (2005).

19. Sun, L. et al. Re-emerging superconductivity at 48 kelvin in iron chalcogenides. Nature 483, 67-69 (2012).

20. Xi, X. X. et al. Signatures of a pressure-induced topological quantum phase transition in BiTeI. Phys. Rev. Lett. 111, 155701 (2013).

21. Tissen, V. G. et al. Pressure dependence of superconducting critical temperature and upper critical field of $2 \mathrm{H}-\mathrm{NbS}_{2}$. Phys. Rev. B 87, 134502 (2013).

22. Suderow, H., Tissen, V. G., Brison, J. P., Martínez, J. L. \& Vieira, S. Pressure Induced Effects on the Fermi Surface of Superconducting $2 \mathrm{H}-\mathrm{NbSe}_{2}$. Phys. Rev. Lett. 95, 117006 (2005).

23. Pan, X-C. et al. Pressure-driven dome-shaped superconductivity and electronic structural evolution in tungsten ditelluride. Nat. Commun. 6, 7805 doi: $10.1038 /$ ncomms 8805 (2015).

24. Lifshitz, I. M. Anomalies of electron characteristics of a metal in the high pressure region. Sov. Phys. JETP 11, 1130-1135 (1960).

25. Liu, C. et al. Evidence for a Lifshiz transition in electro-doped iron arsentic superconductors at the onset of superconductivity. Nat. Phys. 6, 419-423 (2010).

26. Debessai, M., Matsuoka, T., Hamlin, J. J. \& Schilling, J. S. Pressure-induced superconducting state of europium metal at low temperatures. Phys. Rev. Lett. 102, 197002 (2009).

27. Mao, H. K., Xu, J. \& Bell, P. M. Calibration of the ruby pressure gauge to 800 kbar under quasi-hydrostatic conditions. J. Geophys. Res. 91, 4673-4676 (1986).

\section{Acknowledgements}

We thank V. Sidorov for helpful discussions. The work was supported by the NSF of China (Grant Nos 91321207 and 11427805), 973 projects (Grant Nos 2011CBA00100 and 2010CB923000) and the Strategic Priority Research Program (B) of the Chinese Academy of Sciences (Grant Nos XDB07020300 and XDB07020100).

\section{Author contributions}

D.K., Y.Z. and L.S. performed high-pressure resistance, a.c. susceptibility, magnetoresistance and Hall measurements. C.Y. and Y.S. grew the single crystals. Y.Z., D.K., W.Y., S.J., A.L. and K.Y. carried out high-pressure X-ray diffraction measurements. L.S., Q.W., G.Z., Y.Z. D.K. and Z.Z. wrote the paper. All the authors analysed the data and discussed the results.

\section{Additional information}

Competing financial interests: The authors declare no competing financial interests.

Reprints and permission information is available online at http://npg.nature.com/ reprintsandpermissions/

How to cite this article: Kang, D. et al. Superconductivity emerging from a suppressed large magnetoresistant state in tungsten ditelluride. Nat. Commun. 6:7804 doi: $10.1038 /$ ncomms8804 (2015).

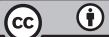

This work is licensed under a Creative Commons Attribution 4.0 International License. The images or other third party material in this article are included in the article's Creative Commons license, unless indicated otherwise in the credit line; if the material is not included under the Creative Commons license, users will need to obtain permission from the license holder to reproduce the material To view a copy of this license, visit http://creativecommons.org/licenses/by/4.0/ 